\title{
Multimodel System Identification Based on New Fuzzy Partitioning Similarity Measure
}

\author{
Abdelhadi Radouane, Fouad Giri, Abdessamad Naitali, Fatima Zahra Chaoui
}

\begin{abstract}
The problem of identifying unstructured nonlinear systems is generally addressed on the basis of multi-model representations involving several linear local models. In the present work, local models are combined to get a global representation using incremental fuzzy clustering. The main contribution is a novel vector similarity measure defined in the System Working Space (SWS) that combines the angular deviation and the usual Euclidean distance. Such a combination makes the new metric highly discriminating leading to a better partitioning of the operating space providing, thereby, a higher accuracy of the model. The developed partitioning method is first evaluated by performing linear local model (LLM) based identification of a academic benchmark multivariable nonlinear system. Then, the performances of the identification method are evaluated using experimental tropospheric ozone data. These evaluations illustrate the supremacy of the new method over the standard Euclidian-distance based partitioning approach.

Keywords: MIMO Nonlinear Systems, System Identification, Local Linear Models, Fuzzy Clustering, Similarity Measure, Angular Deviation, Weighted Least-Squares (WLS).
\end{abstract}

\section{INTRODUCTION}

$\mathrm{N}$ onlinear system identification is of major importance in control and forecasting system design. So far, several identification approaches have been developed to deal with nonlinear systems. The various approaches mainly differ on the model structures used to capture the system nonlinear dynamics. In this respect, one can mention the block-oriented nonlinear model approach [15],[20], [27], the nonlinear state space model approach [9], the Nonlinear System Identification using Neural Networks [37] and the Multi-Model (MM) approach involving LLMs [11], [18], [32], [20], [4]- [6][29]. In this paper, the emphasis is put on the MM approach that consists in determining a collection of Local Linear Models (LLMs) representing the linear behaviour of the system around various operating points and combining these LLMs to obtain a unique nonlinear model

Manuscript received on June 26, 2021.

Revised Manuscript received on July 17, 2021.

Manuscript published on July 30, 2021.

* Correspondence Author

Abdelhadi Radouane*, RMI Lab, FST Hassan First University of Settat, Morocco. Email: abdelhadi.radouane@uhp.ac.ma

Fouad Giri, UNICAEN LAC Lab, Caen Normandie University, Caen, France

Abdessamad Naitali, M2PI Lab, ENSAM, Mohammed V University, Rabat, Morocco

Fatima Zahra Chaoui, M2PI Lab, ENSAM, Mohammed V University, Rabat, Morocco

(c) The Authors. Published by Blue Eyes Intelligence Engineering and Sciences Publication (BEIESP). This is an open access article under the CC BY-NC-ND license (http://creativecommons.org/licenses/by-nc-nd/4.0/) approximating well the global behaviour of the system. The combination of the LLMs is a kind of interpolation of these models using e.g. fuzzy tools [42], [39]. That is the problem of $\mathrm{MM}$ identification amounts to select an appropriate structure of the LLMs, determine the best partition of the LLMs in the system working space (SWS) and estimate their parameters. The LLM partitioning and parameter estimation must be performed, not only in order to minimize the output estimation (prediction or simulation) error, but also in order to minimize the number of required local models i.e. to reduce the size of the MM.

The partitioning problem in MM identification consists in: (i) finding the partitioning parameters i.e. the prototypes and covariance matrices; (ii) and estimating the parameters of the local models. These parameters must be determined so that a cost function of the output estimation error is minimized. There are two main types of partitioning strategies. The first is called supervised classification strategy and consists in a priori partitioning the entire product space including (or covering) the input variables; this task can be performed using e.g. grid partitioning [39] or heuristic tree-construction algorithms [41], [25], [28], [32], [33], [30]. The second partition type, referred to as unsupervised classification, is one where partitioning is driven by the distribution of the experimental data. This involves a cost function where a metric is used to discriminate between available data measurements, leading to overlapping fuzzy subdomains (the wider the input spanned space, the wider the validity domain). The point is that the complexity of all supervised classification methods grows rapidly with the number of input variables. This complexity is due to several facts e.g. grid partitioning leading to subdomains of hyper-cubic form and generating useless local subdomains and redundant sub models (i.e. neighbouring models that provide the same behaviour). Furthermore, the non-uniform distribution of the training, i.e. estimation data in the system working space, also contributes to this complexity.

Various cost functions using standard metrics (e.g. Euclidian or Minkowsky) have been used to cope with the partitioning problem at hand. Besides, these cost functions have been performed in product-space clustering (self-organizing) and have been designed in several works [1], [2], [5]

Presently, we are addressing the (fuzzy) partitioning problem for MM identification by developing an unsupervised partitioning method. The developed method involves a learning process using an Incremental Fuzzy Clustering algorithm (IFC) based on a cost function involving a novel similarity metric.

Published By:

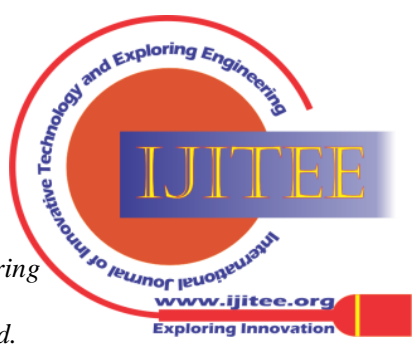


The novelty lies essentially in the combination of Euclidian distance and angular deviation between data vectors. Basically, an incremental fuzzy clustering algorithm amounts to finding expected partitions (while visiting the learning-set), increasing (if necessary) the number of clusters, and simultaneously determining their locations. Doing so, the fuzzy clustering is made much less sensitive to the number and the initial position of the prototypes, compared to non-incremental solutions [12], [10]. Introducing angular deviation in the distance between elements provides the learning algorithm with a higher discriminating capability. Specially, the obtained partitions have the following features: (i) the number of local domains and initial positions of their centers are much better optimized; (ii) the obtained local domains are allowed to take different forms, not only the common ellipsoidal form; (iii) the effects of the curse of dimensionality, which refers to the phenomena that occur when analyzing data in large spaces, are greatly reduced. A preliminary version of this work, limited to mono-input systems, has been presented in the conference paper [36]. The present paper presents the full result corresponding to the multi-input case and providing applications of the identification method to more systems including the three-tank system and the ozone process.

This paper is organized as follows: in Section 2, the identification problem based on MM representation is formulated. In Section 3, the partitioning issue in MM identification is discussed and the proposed solution is presented, the unsupervised fuzzy partitioning based MM identification algorithm is developed and the MM parameter estimation procedure is described. The performances of the developed identification method are illustrated in Section 4 through two case studies. Concluding remarks end the manuscript.

\section{FORMULATION OF THE MULTIMODEL IDENTIFICATION PROBLEM OF NONLINEAR SYSTEMS}

We are considering the class of MISO nonlinear systems that can be represented by the following general nonlinear model:

$$
\begin{gathered}
y(t)=f(\varphi(t))+w(t) \\
\varphi^{T}(t)=\left[z(t-1), z(t-2), \ldots, z\left(t-n_{a}\right), u_{1}(t-1), \ldots,\right. \\
\left.u_{1}\left(t-n_{b}\right), \ldots, u_{l}(t-1), \ldots, u_{l}\left(t-n_{b}\right)\right]
\end{gathered}
$$

where $z(t)$ denotes either the system measured output $\boldsymbol{y}(\boldsymbol{t})$ or an estimated output $\hat{\boldsymbol{y}}(\boldsymbol{t})$ (depending on whether (1) is a Nonlinear AutoRegressive with eXogenous input (NARX) model or Nonlinear Output Error (NOE) model [39]. The function $\boldsymbol{f}($.) is an unknown nonlinear function mapping a subset $\mathbf{\Omega} \subset \mathbf{R}^{\left(\boldsymbol{n}_{a}+\boldsymbol{n}_{b} \times \boldsymbol{l}\right)}$ into $\mathbf{R}$, and $\boldsymbol{w}(\boldsymbol{t})$ is a model error term standing either for output measurement noise or for a bounded modelling error. In the present study, the system model (1) is subject to the following assumptions:

- The function $f($.$) is class C1.$

- The noise $\boldsymbol{w}(\boldsymbol{t})$ is a zero mean sequence of independent random variables.
- The LLM structure parameters $\boldsymbol{n}_{\boldsymbol{a}}$ and $\boldsymbol{n}_{\boldsymbol{b}}$ are upper-bounded by known bounds $\boldsymbol{n}_{\boldsymbol{a}}^{*}$ and $\boldsymbol{n}_{\boldsymbol{b}}^{*}$ respectively.

\subsection{Local Linear Modeling Approach}

The LLM approach consists in partitioning the system working space in a finite (preferably small) number of domains. In each domain, the system is assumed to be well represented by a local linear model (Fig 1). The local models are then combined in a suitable way forming a unique multi-model representation describing well the global behaviour of the system. The local domains are expressed in terms of their centers and associated covariance matrices. Accordingly, the output responses of the LLMs are combined together to form the system output estimate $\hat{\boldsymbol{y}}(\boldsymbol{t})$ as follows:

$$
\hat{y}(t)=\sum_{k=1}^{M} \beta_{k}(\varphi(t)) y_{k}(t)
$$
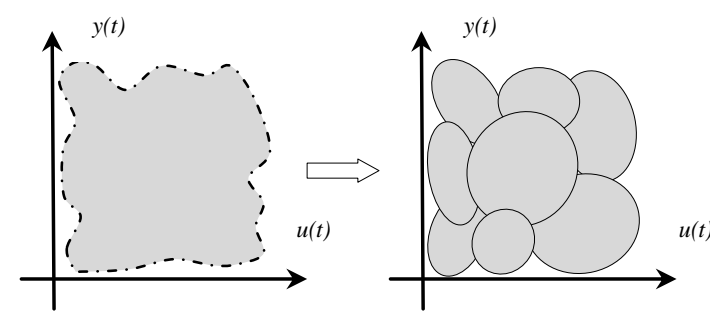

Fig. 1.Partitioning of System Working Space

Where $y_{k}$ is the output of the $k^{\text {th }}$ LLM , $\varphi(t)$ is the system premise variable, and $\boldsymbol{\beta}_{\boldsymbol{k}}($.$) is the Membership Function$ (MSF), subject to some constraints (see equations (6) and (7) hereafter). In equation (3), $\boldsymbol{M}$ is the number of sub-domains and $\boldsymbol{y}_{\boldsymbol{k}}(\boldsymbol{t})$ denotes the output of the $k^{\text {th }}$ LLM and is assumed to be given by the following regression equation:

$$
y_{k}(t)=\varphi_{k}^{T}(t) \theta_{k}+\varepsilon_{k}(t)
$$

Where $\theta_{\boldsymbol{k}}$ and $\varepsilon_{\boldsymbol{k}}$ denote, respectively, the parameter vector and the equation error of the $k^{\text {th }} \operatorname{LLM} ; \varphi_{\boldsymbol{k}}$ is the regressor vector defined as follows:

$$
\begin{aligned}
& \varphi_{k}^{T}(t)=\left[z_{k}(t-1), \ldots, z_{k}\left(t-n_{a}\right), u_{1}(t-1),\right. \\
& \ldots, u_{1}\left(t-n_{b}\right), \ldots, u_{l}(t-1), \ldots, u_{l}\left(t-n_{b}\right)
\end{aligned}
$$

As mentioned earlier, $\boldsymbol{z}_{\boldsymbol{k}}$ designates either the system measured output $y_{k}$ or the estimated output $\hat{y}_{k}$ of the $k^{\text {th }}$ LLM (depending on the nature, NARX and NOE, of the LLM). The model description is completed by the following constraints on the functions $\beta_{k}($.$) , for any \varphi(t)$ at any time $t$ :

$$
\begin{aligned}
& \sum_{k=1}^{M} \beta_{k}(\varphi(t))=1 \\
& \beta_{k}(\varphi(t)) \in[0,1]
\end{aligned}
$$

\subsection{Identification problem formulation}

Considering the local linear modelling equations (2)-(7), the LLM identification problem at hand can be formulated as a constrained multi-objective optimization problem defined as follows: 
- Given a consistent set of input-output estimation data record $Z_{e}^{N} \subset \mathfrak{R}^{N \times(l+1)}$

- Find the minimal System Working Space partitioning parameters $\hat{\Omega}_{\hat{M}}$, including the centers and covariance matrices of the local domains, and find the LLM parameter estimates $\hat{\Theta}_{\hat{M}}$ that minimize the global output errors,

$$
\begin{aligned}
& {\left[\hat{M}, \hat{\Omega}_{\hat{M}}\right]=\arg \min _{M, \Omega_{M}}\left[\begin{array}{c}
M \\
\varepsilon_{G}^{2}\left(Z_{e}^{N}, M, \Omega_{M}, \Theta_{M}\right)
\end{array}\right]} \\
& \hat{\Theta}_{\hat{M}}=\arg \min _{\Theta_{M}} \varepsilon_{G}\left(Z_{e}^{N}, M, \Omega_{M}, \Theta_{M}\right) \\
& \varepsilon_{G}^{2}\left(Z_{e}^{N}, M, \Omega_{M}, \Theta_{M}\right)=\frac{1}{N} \sum_{t=1}^{N}\left(y(t)-\sum_{k=1}^{M} \beta_{k}(\varphi(t)) \varphi_{k}^{T}(t) \theta_{k}\right)^{2}
\end{aligned}
$$

As emphasized by (9), after the SWS is well partitioned, the LLM structures and parameters can be estimated by using local optimization tools such as the weighted least squares (or the principal component analysis), and structure selection criteria such as the Final Prediction Error (FPE). Nevertheless, the main problem which consists in finding the less complex, i.e. short-size partition $\hat{\Omega}_{\hat{M}}$ that matches the global behaviour of the system is still a challenging problem. In summary, multi-model identification consists of answering the following questions:

- What is the optimal number $\hat{\boldsymbol{M}}$ of sub-domains of SWS?

- What common structure should be chosen for local models ( $\hat{\boldsymbol{n}}$ order of LLMs)?

- How should one build the membership functions

$\beta_{k}($.$) delineating the sub-domains?$

- How should the parameters $\hat{\Theta}_{\hat{M}}$ of the local models be determined?

\subsection{On the partitioning issue in Multi-Model identification}

Partitioning in system identification consists in finding the locations of the different local domains within the system working space. It can be done using a variety of techniques that can be divided into two broad categories. A first category uses the strategy of a supervised learning where the partitioning of the SWS is constantly controlled by the user, according to rules provided from information a priori on the behavior of the system. These include: grid partitioning, decision tree-based partitioning [47], partitioning based on non-optimal construction algorithms with increasing heuristic strategies [33], partitioning based on the Johansen and Foss algorithm [25]. The problem with this category of techniques is that one can obtain sub-domains that are never or rarely visited, which makes the modeling unnecessarily heavier [23]. The second category implements the techniques of unsupervised learning (Clustering or self-organization) where the research of sub-domains (clusters) is guided by data collected themselves "data-driven". Partitioning is based on fuzzy classification, partitioning based on neural networks, or partitioning based on neuro-fuzzy techniques [3]. We can add the grouping technique where we start with a large number of small local models and as learning progresses, local models are merged to obtain an optimal structure. With these last techniques, it is certain that the results of the modeling will be much more optimized than those of the first category, but provided that the signals chosen for the excitation are persistent ie they allow to sweep and sufficiently the SWS. They must have enough amplitudes and frequencies appropriate to excite all the interesting modes of the system.

When the transitions in the system working space between the local domains are rather softer than hard, unsupervised fuzzy learning may be an effective tool for data partitioning [21], [8], [19], [23], [4]. Unsupervised learning is data distribution driven clustering method. It consists in seeking the true local domains (clusters) i.e. regimes of a system. When the number of clusters is assumed to be a priori known, the initial positions of the clusters prototypes can be boosted by using an appropriate technique for e.g. see [45], [35], [7]. If the number of subdomains within the SWS is unknown beforehand then standard fuzzy clustering tools, such as fuzzy-c-means (FCM) and Gustafson-Kessel (GK), cannot be used directly because the latter necessitate the knowledge of the number of clusters and their initial positions. Many iterative clustering algorithms combining supervised and unsupervised learning have been proposed to determine the number of sub-domains, see e.g.[43] where at each clustering iteration the largest and worst modelled cluster is divided into two new clusters, before recalling the GK classification. Presently, we get rid of the lack of knowledge on the number $\boldsymbol{M}$ of clusters by designing an incremental Fuzzy clustering algorithm involving a new metric combining the usual Euclidean and angular similarity. The new combined similarity metric is defined here after.

\subsection{New measure of similarity combining Euclidian and Angular distance}

Before addressing partitioning, it is necessary to recall the influence of the choice of the metric to use on the shape of the clusters or sub-domains. The usefulness of a distance is to be able to compare the similarities and the differences between two vectors (more generally between two objects). This comparison is highly sought after in several areas of pattern recognition and automatic classification. The choice of distance is a critical issue for multi-variable data mining methods. Indeed, it is at this stage that it is possible for the operator to make best use of the prior information available to him, in order to choose a relevant measure of distance to compare the similarities between the observations. The most commonly used dis-similarity measure is Minkowski's distance. Euclidean distance is the most common of Minkowski distances and it seems to be the most preferred in engineering work. Euclidean distance is the most common of Minkowski distances and it seems to be the most preferred in engineering work. It imposes spherical shapes for clusters, whereas the other Minkowski distances impose parallelepipedic shapes. In general, the shapes of the clusters can be arbitrary and the choice of a given distance inevitably affects the result of Clustering [13]. Other distances are more preferred in data mining applications (Cluster analysis) because they take into account the density of data in each cluster such as Mahalanobis distances [48], which lead to elliptical shapes. First, recall the general definition of similarity measure.

Published By:

Blue Eyes Intelligence Engineering and Sciences Publication

(C) Copyright: All rights reserved.

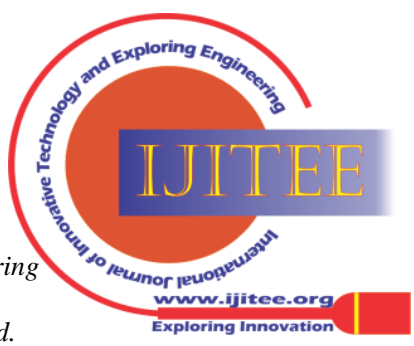


Definition 1 (Similarity measure). A similarity measure $S$ in a subset $\boldsymbol{Z} \subset \mathfrak{R}^{\boldsymbol{n}}$ is defined as a mathematical mapping $\mathbf{Z} \times \mathbf{Z} \rightarrow \mathfrak{R}^{+}$, with the following symmetry, positivity and minimality properties, for all $\boldsymbol{x}, \boldsymbol{y} \in \boldsymbol{Z}$ :

$$
\begin{aligned}
& \mathrm{S}(x, y)=\mathrm{S}(y, x) \\
& \mathrm{S}(x, y) \geq 0 \\
& \mathrm{~S}(x, x) \geq \mathrm{S}(y, x)
\end{aligned}
$$

New similarity measure introduced in this paper is defined as follows:

- Definition 2 (Combined Euclidian - Angular similarity). In the present work the combined Euclidian-Angular similarity $\mathrm{S}_{\boldsymbol{E} \boldsymbol{A}}(\boldsymbol{x}, \boldsymbol{y})$ between two points $\boldsymbol{x}$ and $\boldsymbol{y}$ within the SWS is defined as the root square of the product of the angular deviation $\mathrm{S}_{A}(\boldsymbol{x}, \boldsymbol{y})$ and Euclidian $\mathrm{S}_{\boldsymbol{E}}(\boldsymbol{x}, \boldsymbol{y})$ similarities between vectors $\boldsymbol{x}$ and $\boldsymbol{y}$ :

$$
\begin{gathered}
\mathrm{S}_{E A}(x, y)=\sqrt{\mathrm{S}_{E}(x, y) \times \mathrm{S}_{A}(x, y)} \\
\mathrm{S}_{A}(y, x)=1-d_{A}(y, x) \\
d_{A}(x, y)=\frac{1}{2}\left(1-\frac{x^{T} y}{\|x\| \cdot\|y\|}\right) \\
\frac{x^{T} y}{\|x\| \cdot\|y\|}=\cos (\alpha(x, y)) \in[-1,1] \\
\mathrm{S}_{E}(y, x)=1-d_{E}^{* 2}(x, y) \\
d_{E}^{*}(x, y)=\sqrt{\frac{1}{n}[x-y]^{T} \operatorname{diag}\left\{\left(r_{j}^{-2}\right)_{j=1 . . n}\right\}[x-y]}
\end{gathered}
$$

Where $\boldsymbol{d}_{\boldsymbol{E}}^{*}$ is called normalized Euclidian distance and the $\boldsymbol{r}_{\boldsymbol{j}}$ designates the maximal distance between all points all along the dimension $\boldsymbol{j}$ :

$$
\boldsymbol{r}_{j}=\max _{\boldsymbol{x} \in S W S}\left\{\boldsymbol{x}_{j}\right\}-\min _{\boldsymbol{y} \in S W S}\left\{\boldsymbol{y}_{j}\right\}
$$

$\boldsymbol{x}_{\boldsymbol{j}}$ and $\boldsymbol{y}_{\boldsymbol{j}}$ designate the $\boldsymbol{j}^{\text {th }}$ component of the vectors $\boldsymbol{x}$ and $\boldsymbol{y}$, respectively.

Two vectors representing two distinct objects are said to be similar if and only if they are similar from a point of view of two metrics: the Euclidean distance and the angular distance. Definition 2 is commented upon to emphasize the interest of the new similarity measure. First, note that in fuzzy clustering the Euclidian similarity, when separately considered, allows to distinguish between spherical shape regions (Fig.2). On the other hand, the angular similarity when separately used allows to distinguish between conical shape regions (Fig.2). The new combined similarity measure, as described by Definition 2 , is able to differentiate define, and differentiate between, more complex shapes than just spherical or conical. This power of discrimination seems promising for systems with high nonlinearities.The partitioning example of (Fig.3) illustrates the higher discrimination capability of the combined similarity measure, over Euclidean measure.
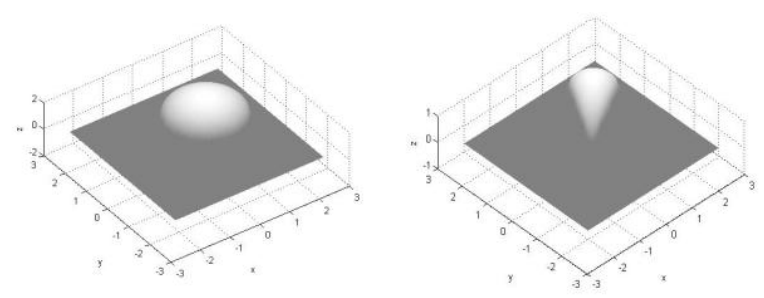

Fig. 2. Equidistant regions to the vector $\left[\begin{array}{lll}1 & 1 & 0\end{array}\right]^{T}$, in the sense of the Euclidian $S_{E}$ (left) and combined $S_{E A}$ (right) similarities respectively.
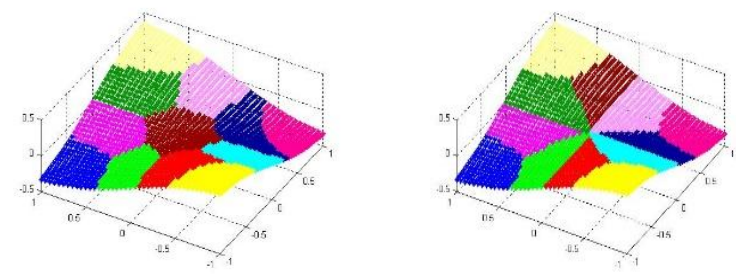

Fig. 3.Partitioning example of nonlinear function $F(x, y)=\frac{x y}{1+x^{2}+y^{2}}$ by using Euclidian (left) and the combined Euclidian-Angular (right) similarity metrics

Now that the combined Euclidian-Angular similarity metric is defined we are ready to define the statements of the unsupervised fuzzy clustering algorithm that will be used in order to seek the size of the MM that captures all the different regimes (or equivalently shapes in the SWS) of the system.

\section{INCREMENTAL FUZZY CLUSTERING (IFC)}

In the present study, the search for the number of sub-models representing the global model in the different zones of the product space of the system is ensured by an Incremental Fuzzy Clustering algorithm. The latter searches the minimal number of clusters that may exist in the training set (as well as prototyping initial positions). The search is performed with respect to a predefined similarity criterion, by applying the following Incremental Fuzzy clustering rule on the training data set.

In the learning phase $(\boldsymbol{t}=\mathbf{1}, \ldots, \boldsymbol{N})$, each $\phi_{t}^{T}$ vector [6] provides information on the distribution of measurements:

$$
\begin{aligned}
\phi_{t}^{T}=\left[-y\left(t-n_{a}\right), \ldots,-y(t-1), u_{1}\left(t-n_{b}\right), \ldots,\right. \\
\left.u_{1}(t-1), \ldots, u_{l}\left(t-n_{b}\right), \ldots, u_{l}(t-1), y(t)\right]
\end{aligned}
$$

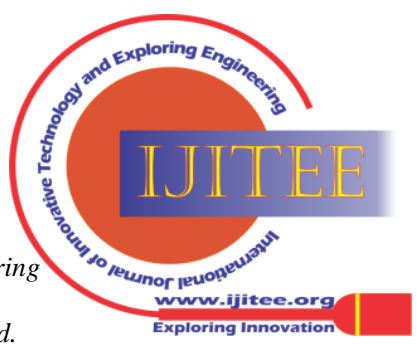




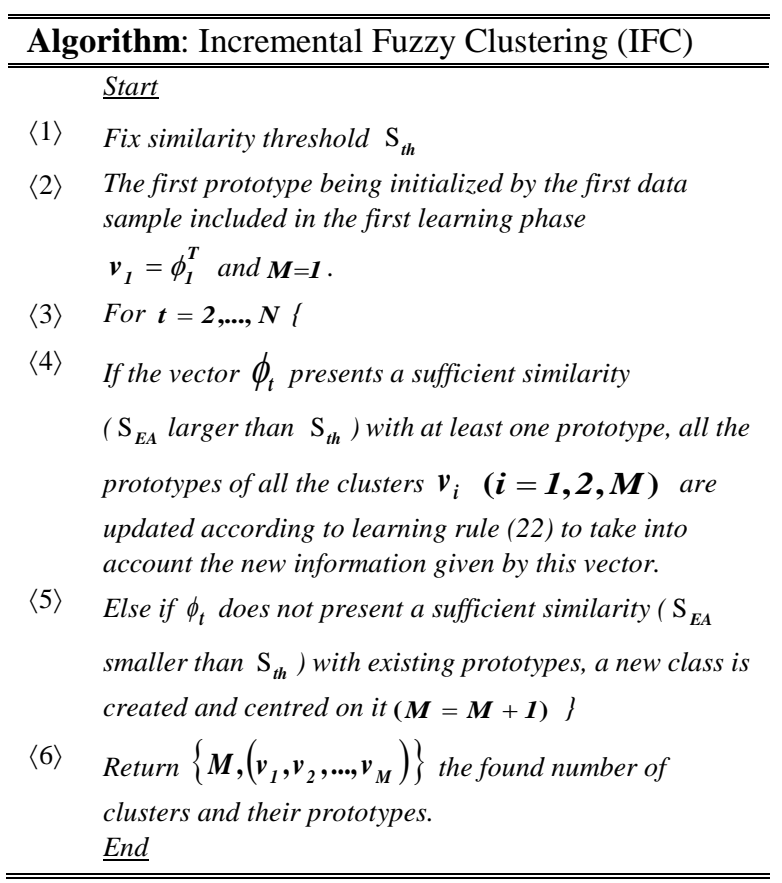

$$
v_{i}^{(\text {new })}=\frac{n_{i, t}}{n_{i, t+1}} v_{i}^{(\text {old })}+\frac{\mathrm{S}_{E A}\left(v_{i}^{(\text {old })}, \phi_{t}\right)}{n_{i, t+1}} \phi_{t}
$$

Where $\boldsymbol{n}_{\boldsymbol{i}, \boldsymbol{t}}$ is the fuzzy cardinality of the cluster $\boldsymbol{i}$ at the iteration $\boldsymbol{t}$ :

$$
n_{i, t}=\sum_{k=1}^{t} S_{E A}\left(v_{i, t}, \phi_{k}\right)
$$

The competitive learning rule (22) can be seen as an adaptation of the general learning rule 'winner takes all' [22]. This provides a simple and effective way to exploit the information given by each new observation $\phi_{t}$ during the learning process. In this step, the similarity threshold $S_{t h}$ is fixed at a given value, and the IFC algorithm provides the number $\boldsymbol{M}$ of clusters (or subdomains) existing in the operating space and their initial centers $\left\{v_{1}^{0}, v_{2}^{0}, \ldots, v_{M}^{0}\right\}$.

\subsection{Unsupervised fuzzy partitioning based Multi Model identification algorithm}

As pointed out earlier in this section, the problem of lack of knowledge of the number $\boldsymbol{M}$ of submodels and their initial prototypes is solved by the IFC algorithm, where partitioning is performed by the use of the combined similarity metric. The obtained partitioning $\hat{\Omega}_{\hat{M}}$ is optimized using the standard algorithm Fuzzy Means Clustering (FCM). The topological properties of the obtained clusters, i.e. the centers and covariance matrices estimates, are then used to construct Members Ship Functions (MSFs) which, in turn, are used to estimate the MM parameter vector $\hat{\Theta}_{M}$ by using the Weighted Least-Squares (WLS) minimization algorithm.

The distance measurement to be used in the FCM optimization algorithm is also based on a combination of distances commonly used in pattern recognition [40], specifically; the used clustering distance is a linear combination of the Euclidian distance (19) and the angular deviation (16), i.e.

$$
d_{E A}=\kappa d_{E}^{*}+(1-\kappa) d_{A}
$$

For some scalar

$$
0 \leq \kappa \leq 1
$$

Bearing in mind the fuzzy clustering topics introduced above, the Skelton of the whole process of the proposed unsupervised fuzzy partitioning, based MM identification algorithm is shown in Figure 3. It is seen that the general identification scheme consists of three main iterative steps, namely: (i) clustering step, carried out by the IFC that provides the number $\boldsymbol{M}$ of sub-domains; (ii) sub-domains' optimization and parameter estimation; (iii) model validation according to prespecified user's criteria. In this algorithm, all possible models with structures, ranging from the minimal to the maximal size, are identified and tested. For clarity, the implementation details of FCM algorithm is summarized in Appendix A1.

The Multi Model identification algorithm based on unsupervised fuzzy clustering begins by initializing the order of the searched LLMs. Then, at each order increment, the similarity threshold $S_{t h}$ is changed according to (28a) and the IFC algorithm is executed. Then, the optimal number $\hat{\boldsymbol{M}}$ of clusters and their initial prototypes are obtained, and the optimization of the partitioning of the SWS is performed using the fuzzy c-means (FCM) clustering algorithm [8]. This series of tasks is repeated until the constructed model successfully passes the validation test. Once the model candidates are built up, the best model in the sense of accuracy/complexity is selected based on the Akaike's Final Prediction Error (FPE) criterion [33]:

$$
\begin{aligned}
& F P E=N \ln (I(\theta))+N \ln \left(\frac{N+n_{p}}{N-n_{p}}\right) \\
& I(\theta)=\frac{1}{N} \sum_{k=1}^{N}(y(k)-\hat{y}(k))^{2}
\end{aligned}
$$

Where $\boldsymbol{N}$ is the number of data samples, $\boldsymbol{n}_{\boldsymbol{p}}$ is the number of effective parameters, and $\boldsymbol{I}(\theta)$ is the loss function.

\subsection{Update of the similarity measure threshold}

The similarity threshold $S_{t h}$ takes values in the interval $\left[S_{\min }, S_{\max }\right]$ where $S_{\min }$ and $S_{\max }$ are a priori determined using a conventional extremum search procedure in the operating space of the system. During the cluster search, the similarity threshold is tuned online as the learning process progresses. Presently, the following iterative law, that reduces the threshold during the learning process, is proposed (see also Figure 4). At each iteration $\boldsymbol{i}_{s}=\mathbf{1}, \mathbf{2}, \ldots, \boldsymbol{i}_{\text {smax }}$, the threshold similarity $S_{t h}^{\left(i_{s}\right)}$ is updated as follows:

$$
\begin{aligned}
& \mathrm{S}_{t h}^{\left(i_{s}\right)}=\mathrm{S}_{\max }\left[1-\left(\frac{\mathrm{S}_{\text {max }}-\mathrm{S}_{\min }}{\mathrm{S}_{\max }}\right) e^{-\delta \times\left(i_{s}-1\right)}\right] \\
& \delta=\frac{1}{i_{S \max }} \ln \left(100 \frac{\mathrm{S}_{\text {max }}-\mathrm{S}_{\min }}{\mathrm{S}_{\text {max }}}\right)
\end{aligned}
$$

With $\delta>\mathbf{0}$ is a parameter controlling step learning.

Published By:

Blue Eyes Intelligence Engineering and Sciences Publication

(C) Copyright: All rights reserved.

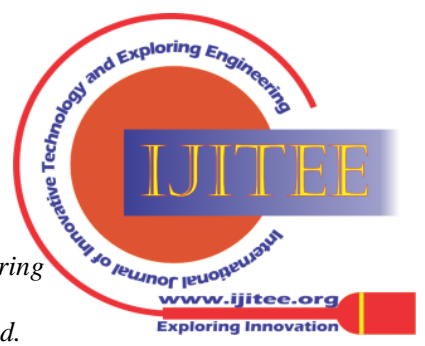


It depends on the relative extent of similarity measure and maximum number of iterations $\left(\boldsymbol{i}_{s_{\max }}\right)$ fixed by the user.

\subsection{Multi-Model Parameter Estimation}

Given the centers and the dispersions of the obtained clusters, the classification of all elements in the SWS can be performed by seeking for each prototype the elements with the highest membership degree. The MM parameter estimation consists in finding the best estimates of the parameters i.e. partitioning properties of the MSFs and the parameters of the LLMs. Presently, these parameters are estimated by exploiting the statistical properties of the clusters and using the WLS algorithm as a local minimization tool.

\section{a) Construction of the MSFs}

In the fuzzy approach, the membership function may be triangular, trapezoidal, Gaussian or sigmoid. In this work, Gaussian MSFs are used which. Each of them is characterized by a mean vector $\boldsymbol{v}_{\boldsymbol{i}}(\boldsymbol{i}=\mathbf{1}, \ldots, \boldsymbol{M})$ and a dispersion matrix $\sigma_{\boldsymbol{i}}$ and is defined as follows [44], [1]:

$$
\beta_{i}(\varphi(t))=\exp \left(-\frac{1}{2}\left(\varphi(t)-C_{i}\right)^{T} Q_{i}^{-1}\left(\varphi(t)-C_{i}\right)\right)
$$

Where $\boldsymbol{C}_{\boldsymbol{i}}$ and $\boldsymbol{Q}_{\boldsymbol{i}}$ designate the center and the dispersion matrix of the first $\boldsymbol{n}$ components of the argument (i.e. vector $\varphi_{t}$ ) belonging to the cluster (i). More specifically, one has:

$$
\begin{aligned}
& \boldsymbol{C}_{\boldsymbol{i}}=\boldsymbol{v}_{\boldsymbol{i}}\left(\mathbf{1}: \boldsymbol{n}_{\varphi}\right) \\
& \boldsymbol{Q}_{\boldsymbol{i}}=\left[\boldsymbol{F}_{\boldsymbol{i}}\left(\mathbf{1}: \boldsymbol{n}_{\varphi}, \boldsymbol{1}: \boldsymbol{n}_{\varphi}\right)\right]^{-1} \\
& \boldsymbol{n}_{\varphi}=\boldsymbol{n}_{\boldsymbol{a}}+\boldsymbol{I} \times \boldsymbol{n}
\end{aligned}
$$$$
F_{i}=\frac{\sum_{k=1}^{N s}\left(u_{k i}\right)^{m}\left(\phi_{k}-C_{i}\right)\left(\phi_{k}-C_{i}\right)^{T}}{\sum_{k=1}^{N s}\left(u_{i k}\right)^{m}}
$$

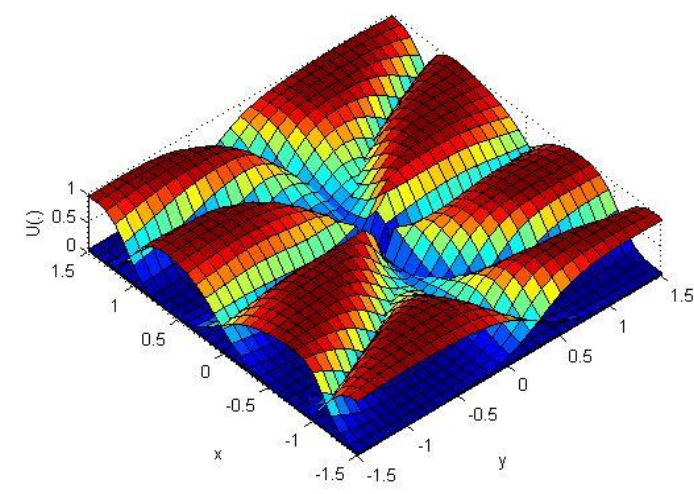

Fig. 4.Example of membership degree function of multi-models

Where $\boldsymbol{F}_{\boldsymbol{i}}$ is the covariance matrix of the set of elements forming the cluster (i), and $\boldsymbol{u}_{\boldsymbol{k} \boldsymbol{i}}$ is the degree of membership of the vector $\phi_{k}$ in cluster (i) of center $\boldsymbol{C}_{\boldsymbol{i}}$ (see Fig. 4).

\section{b) LLM parameter estimation}

Given the properties of the cluster's MSFs, the parameter estimates $\hat{\Theta}$ of MM are estimated using the Weighted Least Squares (WLS) algorithm:

$$
\begin{aligned}
& \hat{\Theta}=\arg \min _{\Theta} J\left(Z_{e}^{N}, \Theta\right) \\
& J\left(Z_{e}^{N}, \Theta\right)=\sum_{t=1}^{N_{s}}\left[y(t)-\Psi(t)^{T} \Theta\right] \\
& \Theta=\left[\theta_{1}^{T} \ldots \theta_{M}^{T}\right]^{T} \\
& \Psi(t)^{T}=\left[\begin{array}{lll}
\beta_{1}(\varphi(t)) \varphi(t)^{T} & \ldots & \beta_{M}(\varphi(t)) \varphi(t)^{T}
\end{array}\right] \\
& \hat{\Theta}=(R)^{-1} \times\left(\sum_{t=1}^{N} \Psi(t) y(t)\right) \\
& R=\sum_{t=1}^{N} \Psi(t) \Psi(t)^{T}
\end{aligned}
$$

It is well known that the WLS estimate converge to its true value if the matrix $\boldsymbol{R}$ full rank which is guaranteed persistently exciting input signals are used in the identification experiment.

Algorithm: Multi Model identification algorithm based on unsupervised fuzzy clustering

$\underline{\text { Start }}$

$\langle 1\rangle \quad$ Acquire the experimental input / output data of the system by respecting the identification protocols.

$\langle 2\rangle \quad$ Initialize the order value of the sub models ( $\boldsymbol{n}:=\mathbf{1})$

$\langle 3\rangle \quad$ Determine the extent of the value of the similarity measure, then initialize the value of its threshold ( $\left.\left[\mathrm{S}_{\min }, \mathrm{S}_{\max }\right], \mathrm{S}_{t h}:=\mathrm{S}_{\min }\right)$

$\langle 4\rangle \quad$ Use the IFC algorithm to detect the number " $\boldsymbol{M}$ " of sub models which can represent the system globally

$\langle 5\rangle \quad$ Call the FCM algorithm to optimize the partitioning of the system workspace

$\langle 6\rangle \quad$ Construct the functions of the degrees of appearance of the sub models and their parameters

$\langle 7\rangle \quad$ Test the combination of models obtained, by calculating the RMS and $\boldsymbol{P F E}$ indicators

<8 If the results are not satisfactory and if the similarity measurement threshold value has not yet reached $\mathrm{S}_{\max }$, update the similarity measurement threshold value $\left(\mathrm{S}_{t h}:=\mathrm{S}_{t h}+\delta\right)$ and go to $\langle 4\rangle$

$\langle 9\rangle$ Else

Increase the value of the order of the multi-models $(\boldsymbol{n}:=\boldsymbol{n}+\mathbf{1})$

$\langle 10$ If the maximum permissible value of the order has not yet been reached, (if ( $\left.\boldsymbol{n}<\boldsymbol{n}_{\max }\right)$ go to $\langle 3\rangle$

¿11 Else

> According to the RMS and PFE criteria, choose the best partitioning and obtain the optimal values of the number $\boldsymbol{M}$ of sub models and the value $\boldsymbol{n}$ of the corresponding order End

\subsection{Identification method assessment}

The multi-model identification method based on fuzzy partitioning that we have just described, will now be evaluated through applications to multivariable nonlinear systems.

Published By:

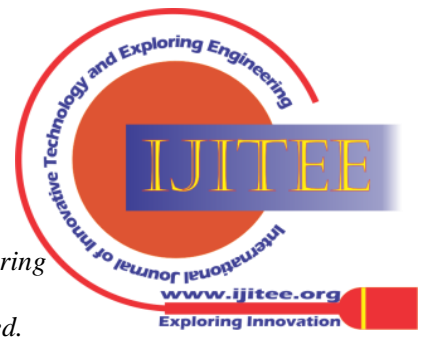


These include the MIMO academic reference in (Narendra, 1990) and the phenomenon of pollution by tropospheric ozone [28]. In any case, the selection of the best multi-model partitioning is performed using the following root mean square (RMSE):

$$
R M S E_{d B}=20 \log _{10}\left(\sqrt{\frac{\sum_{t=1}^{N}(y(t)-\hat{y}(t))^{2}}{\sum_{t=1}^{N} y(t)^{2}}}\right)
$$

\section{RESULT OF SIMULATION AND DISCUSSION}

\subsection{Multi-Model Identification Of Narenda's Mimo Benchmark}

Narenda's MIMO benchmark [31][26] is a two-input two-output nonlinear dynamical system. The two outputs $y_{1}, y_{2}$ are related to the two inputs $\boldsymbol{u}_{1}, \boldsymbol{u}_{2}$ by the following input-output equations:

$$
\left\{\begin{array}{l}
y_{1}(t+1)=\frac{y_{1}(t)}{1+y_{2}^{2}(t)}+u_{1}(t) \\
y_{2}(t+1)=\frac{y_{1}(t) y_{2}(t)}{1+y_{2}^{2}(t)}+u_{2}(t)
\end{array}\right.
$$

Where the input signals take values in the following interval: $\boldsymbol{u}_{\mathbf{1}}(\boldsymbol{t}), \boldsymbol{u}_{\mathbf{2}}(\boldsymbol{t}) \in[-\mathbf{1}, \mathbf{1}]$. The input-output data used in the identification process are collected on the system being excited with Amplitude modulated Pseudo-Random Binary Signals (APRBS). The identification data set of size 600 is divided in two segments: the first 450-length data are used for estimation of the multiple model parameters; the remaining 150-length data are used in model validation. To determine the optimal dimension of the regression vector, the FPE is used. To this end, the effective number of parameters $\boldsymbol{n}_{\boldsymbol{p}}=\boldsymbol{n}_{\boldsymbol{a}}+\boldsymbol{I} \times \boldsymbol{n}_{\boldsymbol{b}}$ is considered and the order $\left(\boldsymbol{n}_{\boldsymbol{a}}=\boldsymbol{n}_{\boldsymbol{b}}=\boldsymbol{n}\right)$ of the regression vector is tuned from 1 to 4 , using both metrics (the Euclidean and the new combined metric). The validation results, based on the criterion FPE, show that the optimal value, for the two output variables, corresponds to $\hat{\boldsymbol{n}}=\mathbf{2}$, see Appendix A2, Fig.14-17.

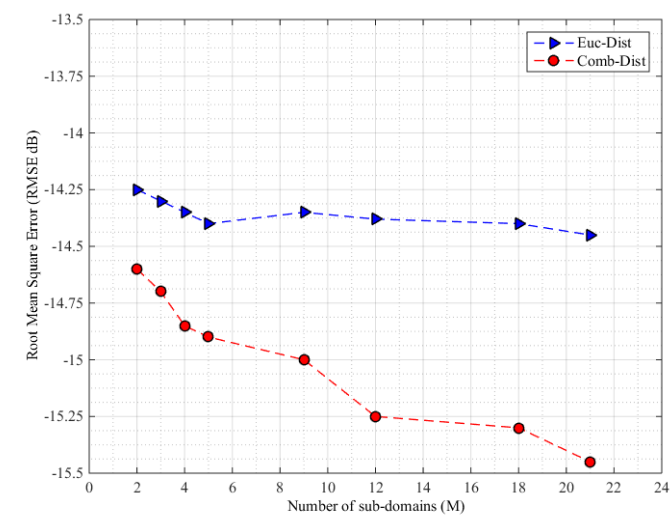

Fig. 5.Estimation of output $y_{1}(t)$ using Euclidean metric (Euc_Dist) and combined metric (Com_Dist), with ordre

$$
\hat{n}=2, R M S E_{d B}=f(M)
$$

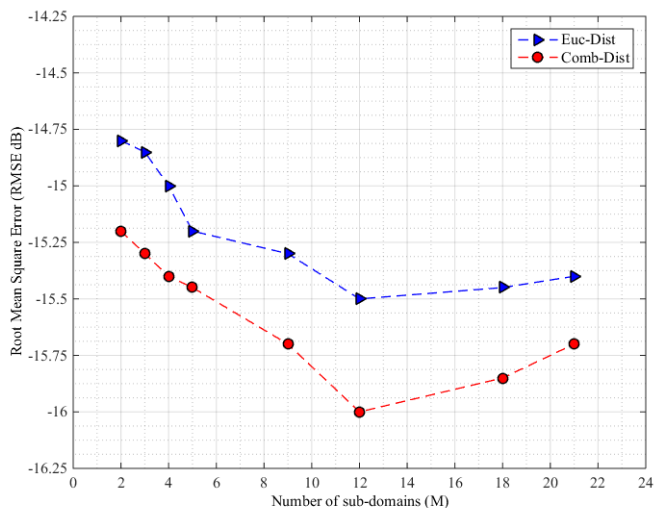

Fig. 6.Validation of output $y_{1}(t)$ using Euclidean metric (Euc_Dist) and combined metric (Com_Dist), with ordre $\hat{n}=2, R M S E_{d B}=f(M)$

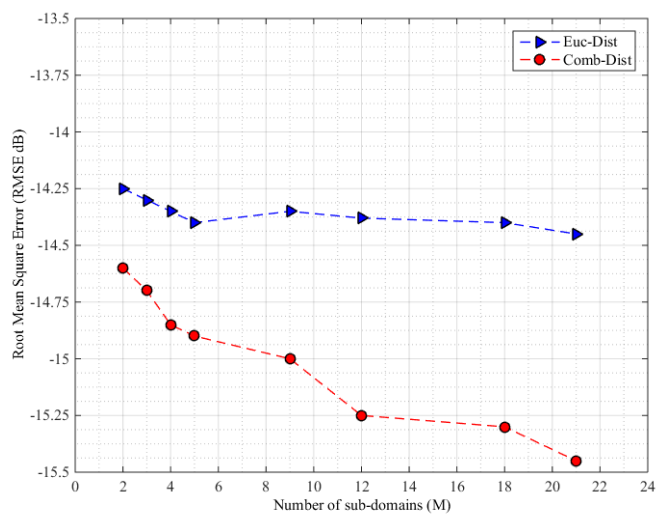

Fig. 7.Estimation of output $y_{2}(t)$ using Euclidean metric

(Euc_Dist) and combined metric (Com_Dist), with ordre $\hat{n}=2, R M S E_{d B}=f(M)$

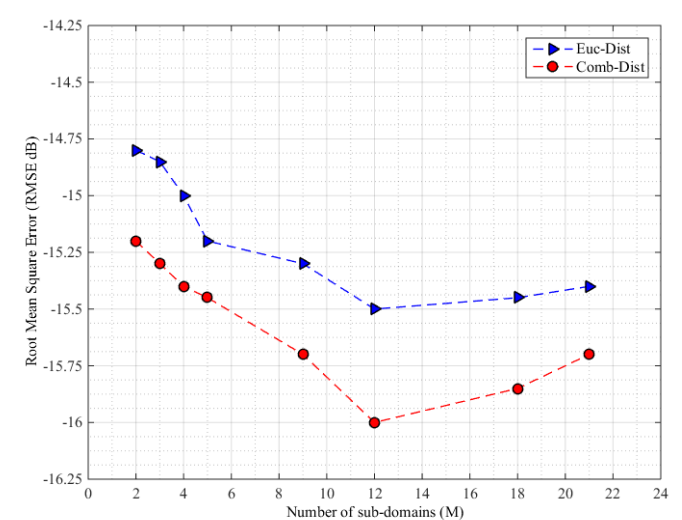

Fig. 8.Validation of output $y_{2}(t)$ using Euclidean metric (Euc_Dist) and combined metric (Com_Dist), with ordre $\hat{n}=2, R M S E_{d B}=f(M)$

Comparing the results obtained with the two metrics, especially during the validation test, we note the importance of the combined metric compared to the Euclidean metric.

Published By:

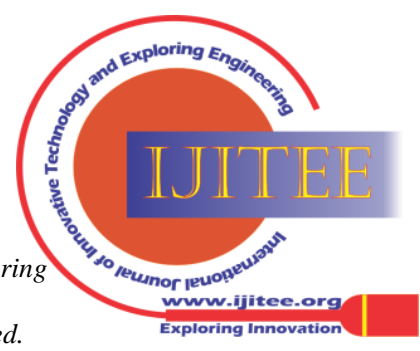


For example, for a quality of RMSE $=-15.5 \mathrm{~dB}$ of the two outputs $\boldsymbol{y}_{1}(\boldsymbol{t})$ (Appendix A2, Fig.15) and $\boldsymbol{y}_{2}(\boldsymbol{t})$ (Appendix A2, Fig.17), only $M=5$ sub-domains will be needed for the combined metric, whereas for the Euclidean metric we need at least $\mathrm{M}=12$ sub-domains.

In validation stage of the global model obtained the obtained error rate obtained (RMSE) with the use of the combined metric is much lower than that obtained using the Euclidean metric (see Fig.5-8). With an optimal order $\hat{n}=2$, the optimal number of sub-models is $\mathrm{M}=12$, and the error rate is better with the new metric than with the Euclidean metric, see Fig. 9.
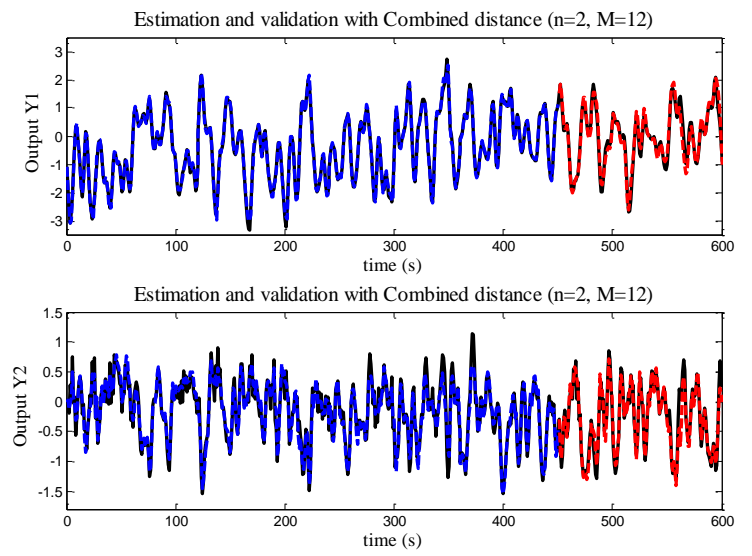

Fig. 9. Measured (black) and estimated (coloured) output using combined metric ( $\hat{n}=2, \hat{M}=12$ ) Identification stage (bleu) and validation stage (red).

4.2. Multi-Model Identification of the tropospheric Ozone Pollution phenomenon

In this example, we are interested in the monitoring of the tropospheric ozone for health and flora safety. Ozone $\left(\mathrm{O}_{3}\right)$ is a

a)

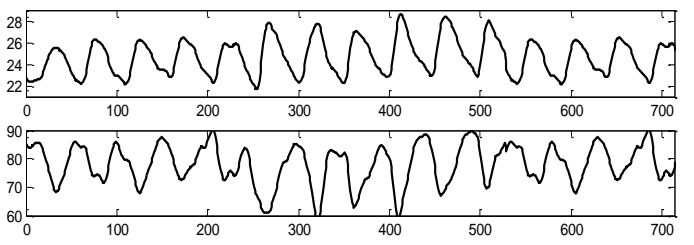

c)

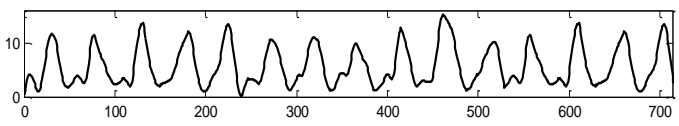

d)

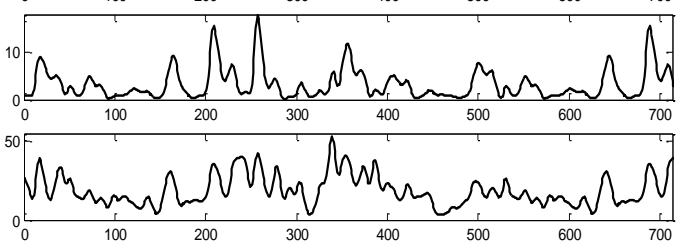

Fig.10. Physical factors affecting on ozone $\mathrm{O3}$ concentration: a) Temperature $\left({ }^{\circ} \mathrm{C}\right)$, b) Humidity (\%), c) Wind speed $(\mathrm{m} / \mathrm{s})$, d) NO $\left(\% \mu \mathrm{g} / \mathrm{m}^{3}\right)$, e) $\mathbf{N O}_{2}\left(\% \mu \mathrm{g} / \mathrm{m}^{3}\right)$ summer pollutant harmful to human health, wildlife and Flora, due to its deep penetration into the lungs. It can cause high concentration inflammation and bronchial hyper-reactivity, Eyes, nose and throat irritations, accompanied by breathing difficulties. The ozone level is an air pollution indicator. It indicates the amount of ozone in the atmosphere. From $180 \mu \mathrm{g} / \mathbf{m}^{3}$, people are aware of the pollution, and from $240 \mu \mathrm{g} / \mathbf{m}^{3}$, a pollution alert is launched. The tropospheric ozone phenomenon is non-linear, multivariable (MISO) and time-varying. Several works has addressed the modelling of the ozone concentration in the ambient area [28], [38], [12]. The selection of influent input variables has been done according to the (hourly) correlation between the output and all input variables [12].

In the present study, the measurement data concerning the period from the first to September 15, 2014 are provided by the "NORMAND AIR" Observatory air quality. The Factors affecting on the concentration of ozone $\mathrm{O}_{3}$ are especially: the temperature, the rate of humidity, the wind speed, and the rates of nitrogen oxide $\mathrm{NO}$ and dioxide $\mathrm{NO}_{2}$. The histogram plots in Fig. 14 confirm this.

The number of input signals is relatively high, $(I=5)$ and with an order $\left(\hat{\boldsymbol{n}}_{\boldsymbol{a}}=\hat{\boldsymbol{n}}_{\boldsymbol{b}}=\mathbf{4}\right)$, the size of regression vectors reached 25 . The problem of the concentration of the fractional distances [17], also called the curse of dimensionality, is displayed. At this level, the contribution of discriminating character of the angular deviation is feeling well and helps to separate observations (regression vectors). The selected input data measurements used to carry out the MM identification algorithm are plotted together in Fig. 10. a)

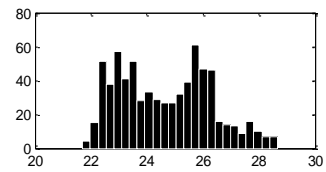

c)

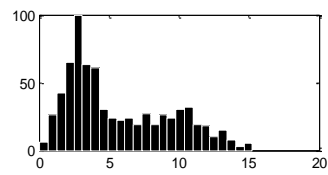

e)

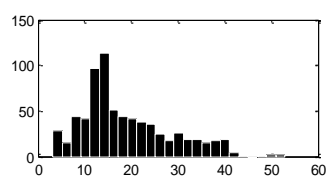

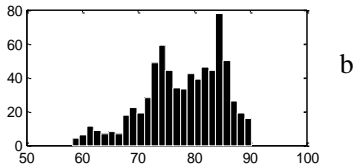

b)

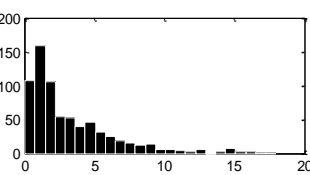

d)

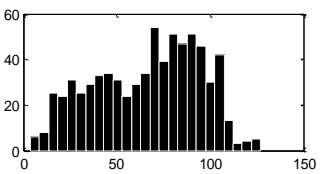

Fig.11. Distribution of the factors influencing the concentration of ozone in the air a) Temperature $\left({ }^{\circ} \mathrm{C}\right)$, b) Humidity (\%), c) Wind speed (m/s), d) NO $\left(\% \mu \mathrm{g} / \mathrm{m}^{3}\right)$, e) $\mathrm{NO}_{2}\left(\% \mu \mathrm{g} / \mathrm{m}^{3}\right)$, f) ozone $\mathrm{O3}\left(\% \mu \mathrm{g} / \mathrm{m}^{3}\right)$

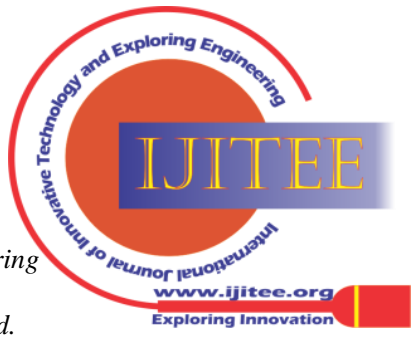




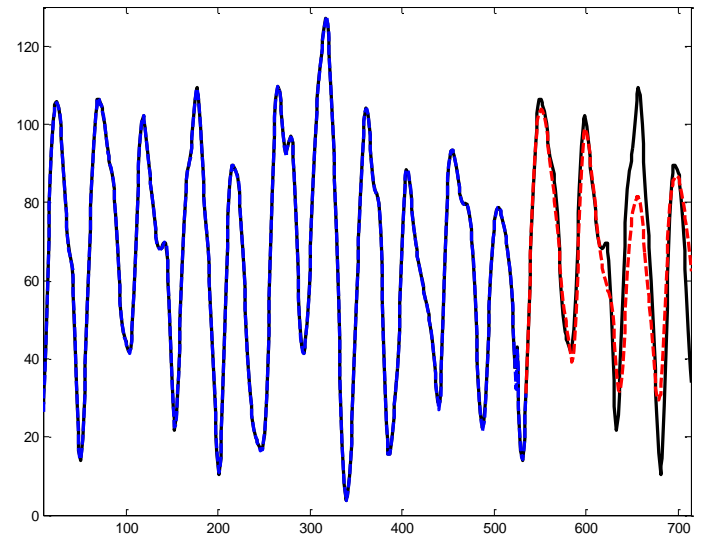

Fig.12. Measured (black) and estimated ozone concentration with Euclidean metric; estimation (blue) and validation

$$
\text { (red) }\left(\hat{n}_{a}=\hat{n}_{b}=4 ; \hat{M}=16\right)
$$

Applying our approach to the prediction of the rate of ozone in the ambient air, we can see that it can be expected at least in the next three days with good precision(Fig. 13). This confirms the interessant relative results obtained by the new metric introduced.

\section{CONCLUSION}

In this work, we have addressed nonlinear dynamical multivariable systems identification using the multi-model approach based on fuzzy classification. A new similarity measure is constructed, by combining Euclidian distance with an angular metric that enjoy higher discriminating capability than standard metrics. Partitioning operating space is carried out by means of two main nested loops. The external loop is devoted to finding local models order while the internal loop is devoted to determining the threshold of similarity measure. Clusters search is performed by the fuzzy learning based FCM algorithm. Finally, the weighting functions are constructed and parameters estimation, of each sub-model, is carried out by least squares (LS) technique. RMSE and PFE criteria are used in model validation. The proposed identification approach is assessed through applications to nonlinear dynamic system benchmarks and to ozone concentration modelling in ambient air. The results obtained, show that, with the combined metric, it is possible to obtain a good compromise between the precision (error rate) and the complexity (number of sub-domains and order of the sub-models) of the model overall obtained, compared to the results obtained with the Euclidean metric alone. This would give more interest to the multimodel identification of the nonlinear dynamic systems.

\section{APPENDIX A1}

FCM algorithm generates a fuzzy partition. This means that each data sample is assigned to each cluster with a certain degree of membership. For each data sample all degrees of membership sum up to 1 (41). And no cluster is completely empty, and no cluster aggregates all data (42).

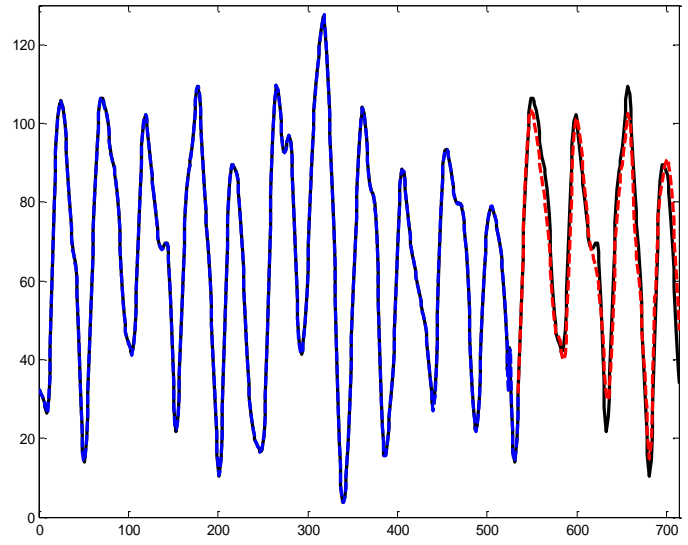

Fig.13. Measured (black) and estimated output signals with metric combining the angular deviation; estimation

(blue) and validation (red) $\left(\hat{n}_{a}=\hat{n}_{b}=4 ; \hat{M}=16\right)$

$$
\begin{aligned}
& \sum_{i=1}^{M} u_{i t}=1 ; \forall t=1, \ldots, N_{s} \\
& 0<\sum_{t=1}^{N_{s}} u_{i t}<N ; \forall i=1, \ldots, M
\end{aligned}
$$

〈2 Initialize:

$>\quad-\quad$ initial centers : $\boldsymbol{V}^{(0)}=\left\{\boldsymbol{v}_{1}{ }^{(0)}, \boldsymbol{v}_{2}{ }^{(0)}, \ldots, \boldsymbol{v}_{M}{ }^{(0)}\right\}$

- Counter of iterations: $\boldsymbol{k}=\mathbf{1}$

<3 Repeat \{

$>\quad$ for $\boldsymbol{i}=1,2, \ldots, M$ and for $\boldsymbol{t}=1,2, \ldots, N_{s}$ Calculate $\boldsymbol{U}=\left[\boldsymbol{u}_{\mathbf{i t}}\right]$ according to (44)

- for $_{\mathbf{i}}=1,2, \ldots, \mathrm{M}$

Update the new centers $\left[\boldsymbol{V}_{\boldsymbol{i}}^{(\boldsymbol{k})}\right]$ according to (45)

- $k=k+1$ \} until $\left(\left\|V^{(k)}-V^{(k-1)}\right\|<\xi\right)$ or $\left(\boldsymbol{k} \geq \boldsymbol{k}_{\max }\right)$

$\langle 4 \quad$ Return $(\hat{V}, \hat{U})$

End

This algorithm is based on the minimization of the objective function (43). Since this functions minimized by clustering techniques is typically nonlinear, FCM algorithm operates iteratively starting from initially chosen clusters

Published By:

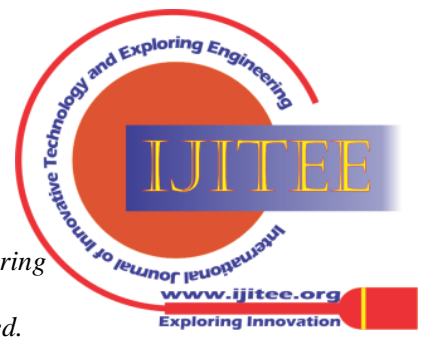




$$
\begin{aligned}
J_{m}(U, V)=\sum_{i=1}^{M} \sum_{t=1}^{N_{s}} u_{i t}^{m} d^{2}\left(\phi_{t}, v_{i}\right) ; \\
\quad i=1, \ldots, M ; t=1, \ldots, N_{s}
\end{aligned}
$$

where for each iteration of the clustering process the membership degree (MSD) of each element to each cluster $\boldsymbol{U}=\left(\boldsymbol{u}_{\boldsymbol{i t}}\right)_{\boldsymbol{i}=1 . . M, \boldsymbol{m}=1 . . N_{s}}$ and the positions of the clusters $\boldsymbol{V}=\left(\boldsymbol{v}_{\boldsymbol{i}}\right)_{\boldsymbol{i}=\mathbf{1 . .} \boldsymbol{M}}$ are updated by (44-45) respectively.

$$
\begin{aligned}
& u_{i, t}=\frac{1}{\sum_{j=1}^{M}\left[\frac{d\left(\phi_{t}, v_{i}\right)}{d\left(\phi_{t}, v_{j}\right)}\right]^{\frac{2}{m-1}}} ; \\
& v_{i}=\frac{\sum_{t=1}^{N}\left(u_{i, t}\right)^{m} \phi_{t}}{\sum_{t=1}^{N}\left(u_{i, t}\right)^{m}}
\end{aligned}
$$

The parameter $\boldsymbol{m}$ controls the fuzziness of the memberships and usually it is set to 2 . For high values of $\boldsymbol{m}$ the algorithm tends to set all the memberships equals meanwhile for $\boldsymbol{m}$ tending to 1 we obtain the K-Means algorithm where the memberships are crisp. The algorithm stops when the prototypes of all the clusters $\left(v_{i} ; \boldsymbol{i}=\mathbf{1} \ldots M\right)$ become almost stable by satisfying: $\left\|\boldsymbol{V}_{i}^{(k)}-\boldsymbol{v}_{i}^{(k-1)}\right\|<\xi$

\section{APPENDIX A2}

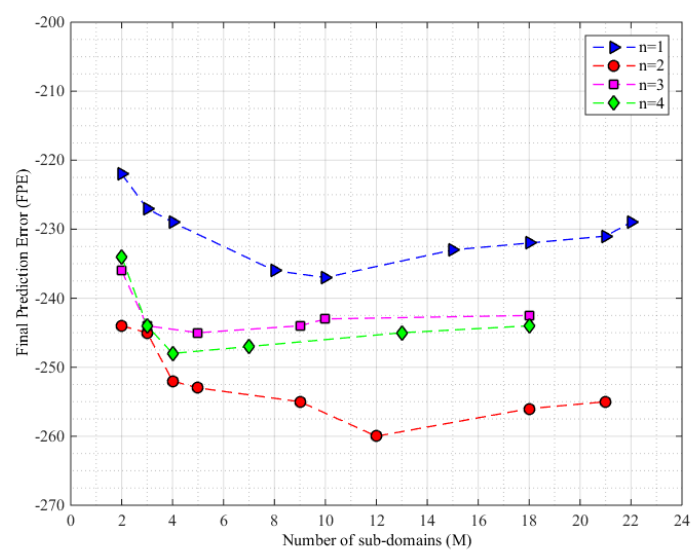

Fig. 10. Validation of output $y_{1}(t)$ using Euclidian metric, $F P E=f(M) \rightarrow \hat{\boldsymbol{n}}=\mathbf{2}$

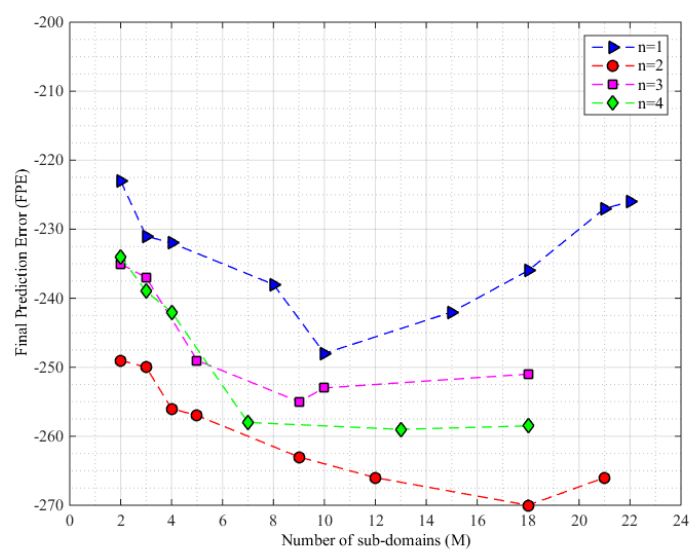

Fig. 11. Validation of output $y_{1}(t)$ using the new combined metric, $F P E=f(M) \rightarrow \hat{n}=2$

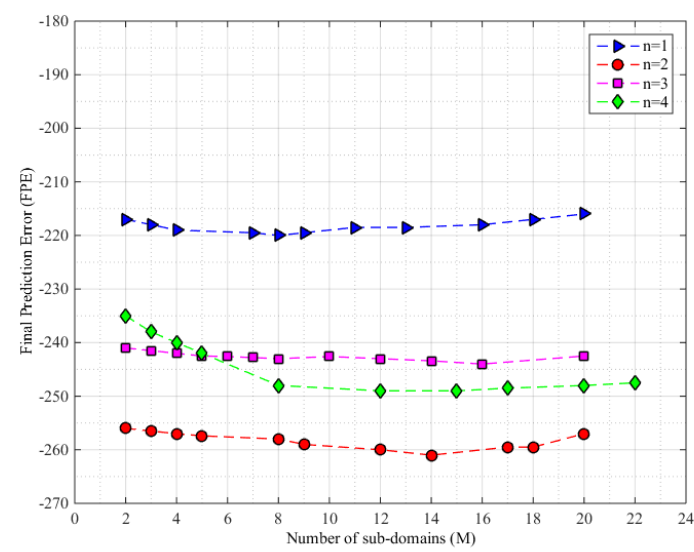

Fig. 12. Validation of output $y_{2}(t)$ using Euclidian metric, $F P E=f(M) \rightarrow \hat{\boldsymbol{n}}=2$

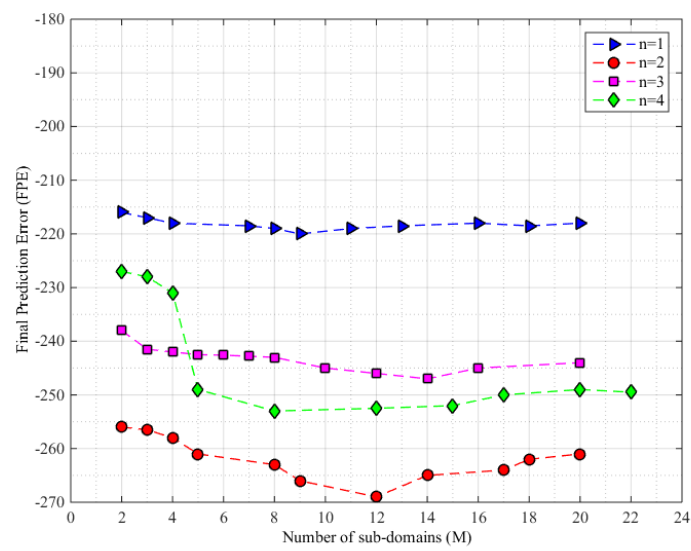

Fig. 13. Validation of output $y_{2}(t)$ using the new combined metric, $F P E=f(M) \rightarrow \hat{\boldsymbol{n}}=2$

\section{REFERENCES}

1. Abonyi J. Feil,B., (2007). Cluster Analysis for Data Mining and System Identification. Birkhauser Verlag AG Berlin.

2. Abonyi J., Babuska R. and Szeifert, F., (2002). Modified Gath-Geva Fuzzy Clustering for Identification of Takagi-Sugeno Fuzzy Models, IEEE Transactions on Systems, Man and Cybernetics,Vol. 32.

3. Abonyi J., Chovan T., Szeifert F., (2001). Identification of Nonlinear Systems using Gaussian Mixture of Local Models. Hungarian Journal of Industrial Chemistry. Vol. 29, pages 134-139.

4. Babuska R., van der Veen P.J., Kaymak U., (2002). Improved covariance estimation for gustafson-kessel clustering. In Proceedings of FUZZY-IEEE, pp:1081-1085.

5. Babuska R. and Verbruggen H. B., (1997). Fuzzy sets methods for local modelling and control. Taylor and Francis.

6. Babuska R., (1996). Modeling and Identification. PhD thesis, Dept. of Control Engineering, Delft University of Technology, Delft, The Netherlands.

7. Bensaid A.M., Hall L.O., Bezdek J.C, Clarke L.P., Silbiger M.L., Arrington J.A., and Murtagh R.F., (1996). Validity-guided (Re) Clustering with applica-tions to imige segmentation. IEEE Transactions on Fuzzy Systems, 4:112 -123.

8. Bezdek J.C., (1981). Pattern Recognition With Fuzzy Objective Function Algorithms. Plenum Press,New York.

9. Billings S. A., (2013). Nonlinear System Identification : NARMAX Methods in the Time, Frequency, and Spatio-Temporal Domains. Chichester, UK:John Wiley \& Sons.

Published By: and Sciences Publication

(C) Copyright: All rights reserved.

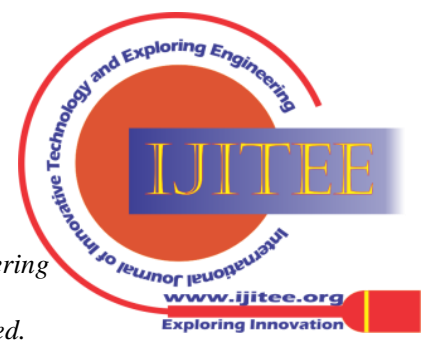


10. Bouroumi A., Limouri M. and Essaïd A., (2000). Unsupervised Fuzzy Learning and Cluster Seeking. Intelligent Data Analysis, Vol. 4 No. 3.

11. Chen S. and Billings S. A., (1989). Representation of non-linea systems: the NARMAX model. Int. Journal of Control, vol. 49, N03.

12. Elayane E., Giri F., Pigeon E., and Massieu J-F., (2010). 24h predictor of the Ozone process for Basse-Normandie region using fuzzy approach. Intelligent Systems (IS), 5th IEEE International Conference.

13. Everitt B.S., Landau S., Leese M. (2011), Cluster Analysis, 5th Edition. John Wiley \& Sons, Ltd.

14. F. He and Y. Yang, "Nonlinear System Identification of Neural Systems from Neurophysiological Signals," Neuroscience, vol. 458, pp. 213-228, 2021, doi: 10.1016/j.neuroscience.2020.12.001.

15. F. Alonge, R. Rabbeni, M. Pucci, and G. Vitale, "Identification and Robust Control of a Quadratic DC/DC Boost Converter by Hammerstein Model," IEEE Trans. Ind. Appl., vol. 51, no. 5, pp 3975-3985, 2015, doi: 10.1109/TIA.2015.2416154

16. Falasconi M., Gutierrez A., Pardo M., Sberveglieri G., and Marco S., (2010). Asability based validity method for fuzzy clustering. Pattern Recognit. ,vol 43,no 4,pp 1292-1305.

17. François D., Wertz V., (2007). The Concentration of Fractional Distances. IEEE Transactions on knowledge and data engineering, vol $19, \mathrm{n}^{\circ} 7$.

18. Gasso K., (2000). Identification des systèmes dynamiques non-linéaires : approche multimodèle. Doctorat de l'institut National Polytechnique de Lorraine, Nancy.

19. Gath, I. and Geva A.B., (1989) .Unsupervised optimal fuzzy clustering IEEE Transactions on Pattern Analysis and Machine Intelligence 7, 773-781.

20. Giri F. and Bai E.-W., (2010). Block-oriented Nonlinear System Identification. Springer, 1 edition.

21. Gustafson D.E. and Kessel V.C., (1979). Fuzzy clustering, with a fuzzy co-variance matrix. In: Proc IEEE. CDC, San Diego, 761-766.

22. Kohonen T., (1990). The self-organizing map. Proceedings of the IEEE.

23. Jakubek S., Keuth N., (2005). A New Training Algorithm for Neuro-Fuzzy Networks. In: Proceedings of the 2nd International Conference on Informatics in Control, Automation and Robotics. Barcelona, Spain.

24. Jain A.K., Murty M.N., Flynn, P.J., (1999). Data Clustering :A Review. ACM Computing Surveys.

25. Johansen T.A. and Foss A.B., ( 1995). Identification of non-linear system structure and parameters using regime decomposition. Automatica, 31.

26. Kroll A., Schulte H., (2014). Benchmark problems for nonlinear system identification and control using Soft Computing methods: Need and overview. Applied Soft Computing 25, pp 496-513

27. Schoukens M. and Tiels K., "Identification of block-oriented nonlinear systems starting from linear approximations: A survey," Automatica vol. 85, pp. 272-292, 2017, doi: 10.1016/j.automatica.2017.06.044.

28. Mourot G., Gasso K., Ragot J., (1999). Modelling of ozone concentrations using a Takagi-Sugeno model. Control Engineering Practice, vol. 7, pp. 707-715.

29. Murray-Smith R. et Johansen, T. A., (1997). Multiple model approaches to modeling and control. Taylor \&Francis, London.

30. Naitali A., Giri F., Radouane A., Chaoui F. Z (2014). Swarm intelligence based partitioning in local linear models identification. ISIC 2014: 843-848.

31. Narendra K., Parthasarathy K., (1990). Identification and control of dynamical systems using neural networks, IEEE Trans. Neural Netw. Pp: 1 4-27.

32. Nelles O. (1997). Orthonormal basis functions for nonlinear system identification with local linear model trees (LOLIMOT). Proceeding of 11th IFAC Symposium on System Identification. Kitakyushu, Fukuoka, Japan.

33. Nelles O., (2001). Nonlinear system identification. Springer-Verlag. Berlin Heidelberg

34. Paduart, J., Lauwers, L., Swevers, J., Smolders, K., Schoukens, J., \& Pintelon, R., (2010). Identification of nonlinear systems using polynomial nonlinear state space models. Automatica, 46(4), 647-656.

35. Pal N. R, Bezdek (1995). On cluster validity for th fuzzy c-means model.

36. Radouane A., Giri F., Naitali A., Chaoui FZ., (2013). Similarity Improvement Using Angular Deviation in Multimodel Nonlinear System Identification. 11th IFAC International Workshop on Adaptation and Learning in Control and Signal Processing. Vol. 11, pp 605-610.

37. Purwar S., Kar I N \& Jha A N (2007) Nonlinear System Identification using Neural Networks, IETE Journal of Research, 53:1, 35-42, IEEE Trans. On Fuzzy Systems Vol. 3, no 3, pp. 370-379

38. Salazar-Ruiz A., Ordieres J.B., Vergara E.P,. Capuz-Rizo S.F, (2008). Development and comparative analysis of tropospheric ozone prediction models using linear and artificial intelligence-based models in Mexicali, Baja California (Mexico) and Calexico, California (US). Environmental Modelling \& Software 23. Pp 1056-1069

39. Shorten R. and Murray-Smith R., (1997). Multiple model approaches to modelling and control, chapter Side-effects of normalizing basis functions in local model networks. Taylor and Francis.

40. Shyjan M., Martial H., (2003). The Optimal Distance Measure for Object Detection. In: IEEE Computer Society Conference on Computer Vision and Pattern Recognition (CVPR'03)

41. Sun C.T, (1994). Rule-base structure identification in an adaptive-network-based fuzzy inference system. IEEE Trans. on Fuzzy Systems, 2(1) pages:64-73.

42. Takagi T.M. and Sugeno M., (1985). Fuzzy identification of systems and its application to modelling and control. IEEE Transactions on Systems, Man and Cybernetics 15 (1), 116-132.

43. Teslic L., Hartmann B., Nelles O., and Škrjanc I., (2011). Nonlinear System Identification by Gustafson- Kessel Fuzzy Clustering and Supervised Local Model Network Learning for the Drug Absorption Spectra Process. neural networks, IEEE transactions.

44. Trabelsi A., Lafont F., Kamoun M. and Enea G., (2004). Identification of nonlinear multivariable systems by adaptive fuzzy Takagi-Sugeno model. International Journal of Computational Cognition. Volume 2, Number 3, Pages 137-153.

45. Windham M.P., (1981). Cluster validity for fuzzy clustering algorithm. Fuzzy Sets and Systems. Vol. 5, pp. 177-185.

46. Xin Liu, Xianqiang Yang \& Xiaofeng Liu (2019): Nonlinear state-space system identification with robust Laplace model, International Journal of Control, DOI:10.1080/00207179.2019.1658134

47. H. Gollee \& K. J. Hunt (1997) Nonlinear modelling and control of electrically stimulated muscle: A local model network approach, International Journal of Control, 68:6, 1259-1288.

48. Mahalanobis, P.C. (1936) On the Generalized Distance in Statistics Proceedings of the National Institute of Science of India, 2, 49-55.

\section{AUTHORS PROFILE}

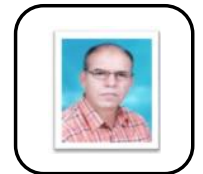

Abdelhadi Radouane, was born in 1969. He received the Agrégation degree in Electrical Engineering from the ENSET of Rabat, Morocco in 1996. In 2017, he obtained a Ph.D in Automatic Control. Since 2018 he joined the FST Settat, University of Hassan First, Morocco as Assistant-Professeur.

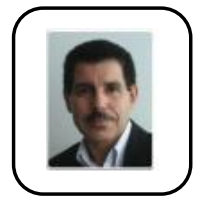

Fouad Giri, received his Ph.D. in Automatic Contro from the Institut National Polytechnique of Grenoble, France, in 1988. He is a Professor at the University of Caen Basse-Normandie, Caen, France and a member of the GREYC Lab (CNRS UMR 6072). He has held visiting research positions at the University of Southern California, the Ruhr University of Bochum (Germany) and the Polytechnic University of Catalunya, Barcelona, Spain. He has held a Vice-Chair position in the IFAC Technical Committee 'Adaptive and Learning Systems' and served as a member of the IFAC Technical Committees 'Modeling, Identification and Signal Processing' and 'Power Plants and Power Systems' and of the IEEE CSS TC 'System Identification and Adaptive Control'. He has been serving as Associate Editor of the IFAC journal 'Control Engineering Practice', of the IEEE Transactionson Control Systems Technology and of the Editorial Board of IEEE CSS conferences. His research interests include nonlinear system identification, nonlinear adaptive control, constrained control, and applications of control theory to power converters, electric machines and power systems. He has co-authored over 250 journal and conference papers and three books.

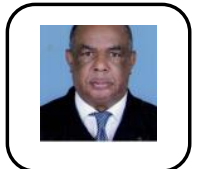

Abdessamad Naitali, was born in Rabat, Morocco, in February 1963. He received his bachelor of education in electrical engineering in 1987 from the ENSET of Rabat (Morocco), the Aggrégation in electrical engineering in 1993 from the ENS of Cachan (France), and his deep up graduate studies diploma in industrial process driving and $\mathrm{PhD}$ in automatic control and industrial informatics from the EMI of the university Mohammed V of Rabat (Morocco) in 2007, respectively.

Published By: 
He worked as a certificate professor from 1987 to 1992 and as an aggregated professor from 1993 to 2010 at the ENSET of Rabat, respectively. In 2013 he was distinguished as an engineering educator by Marquis Who's Who in the world. Now he is an assistant professor at the University of Mohammed V of Rabat.

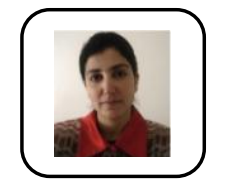

Fatima-Zahra Chaoui, obtained a Ph.D. degree in Automatic Control from the Institut National Polytechnique of Grenoble, France, in 2000. She has spent long-term visits at the Laboratoire d'Automatique de Grenoble and the GREYC, University of Caen, both in France. Since 1995, she has been successively Assistant Professor and Professor at the ENSAM of Rabat, Morocco. 